\title{
Correspondence
}

http://dx.doi.org/10.11646/phytotaxa.174.4.6

\section{A new combination in Asian Clinopodium (Lamiaceae)}

\author{
I.M. TURNER \\ Research Associate, Royal Botanic Gardens Kew, Richmond, Surrey, UK. \\ Email:turner187@btinternet.com
}

Clinopodium umbrosum (M.Bieb.) Koch (1849: 673) was originally described from Georgia. A broad view of this species, which ranges from the Mediterranean to East Asia, has been maintained by some authors employing this name (Cramer 1981, Leblebici 1982, Clement 1999) or one of its homotypic synonyms (e.g. Satureja umbrosa (M.Beib.) Scheele (1843: 577), Keng 1978). However, from the Himalayas eastward one or more different species have been recognised by others. The name C. repens (D.Don) Benth. in Wallich (1830: 66) has been used for the eastern element in two major Chinese floras (Wu \& Hsuan 1977, Li \& Hedge 1994), although it was not noted that this name was first validly used for a South American species, published by Vellozo in 1829 (Stafleu \& Cowan 1988), the year before Bentham transferred Thymus repens Don (1825: 113) to Clinopodium. Clinopodium repens (D.Don) Benth. in Wallich (1830: 66) is therefore an illegitimate later homonym and cannot be used. The next available synonym appears to be Ziziphora javanica Blume (1826: 822), described from Java. As there is no combination in Clinopodium for this basionym, I propose a new combination below:

Clinopodium javanicum (Blume) I.M.Turner, comb. nov. Basionym:-Ziziphora javanica Blume (1826: 822). Type:-INDONESIA. Java, Tjeremai, s.dat., C. Blume 1595 (lectotype, designated by Keng (1969: 45): L (barcode no. L0003817)).

Thymus repens Don (1825: 113) [non T. repens Gilibert (1782: 74), nom. inval., opus utique oppr.]. Clinopodium repens (D.Don) Benth. in Wallich (1830: 66), nom illegit., non C. repens Vellozo (1829: 242). Type:-NEPAL. Suembu, 25 May 1802, F. Buchanan-Hamilton s.n. (holotype: BM (barcode no. BM000778849)).

\section{References}

Blume, C.L. (1826) Bijdragen tot de flora van Nederlandsch Indië (14). Lands Drukkerij, Batavia, pp. 731-850.

Clement, R.A. (1999) Labiatae. In: Grierson, A.J.C. \& Long, D.G. (Eds.) Flora of Bhutan 2(2). Royal Botanic Garden, Edinburgh, pp. 938-1002.

Cramer, L.H. (1981) Lamiaceae (Labiatae). In: Dassanayake, M.D. (Ed.) Revised Handbook to the Flora of Ceylon 3. A.A. Balkema, Rotterdam, pp. 108-194.

Don, D. (1825) Prodromus florae nepalensis. J. Gale, London, 256 pp.

Gilibert, J.E. (1782) [“1781'] Flora lituanica inchoata. Typis S.R.M., Grodno, 308 pp.

Keng, H. (1969) Flora Malesianae Precursores XLVIII. A revision of Malesian Labiatae. Gardens'Bulletin, Singapore 24: 13-180.

Keng, H. (1978) Labiatae. In: van Steenis, C.G.G.J. (Ed.) Flora Malesiana 8(3). Sijthoff \& Noordhoff International Publishers, Alphen aan den Rijn, pp. 301-394.

Koch, K. (1849) Beiträge zu einer Flora des Orientes: Labiatae, Lippenblüthler. Linnaea 21: 639-706.

Leblebici, E. (1982) Clinopodium. In: Davis, P.H. (Ed.) Flora of Turkey 7. Edinburgh University Press, Edinburgh, pp. 329-331.

Li, X. (Hsi-wen) \& Hedge, I.C. (1994) Lamiaceae (Labiatae). In: Wu, Z.-Y., Raven, P.H. \& Hong, D.Y. (Eds.) Flora of China 17. Science Press, Beijing, and Missouri Botanical Garden Press, St Louis, pp. 50-299.

Scheele, A. (1843) Beiträge zur deutschen und schweizerischen Flora. Flora 34: 557-586.

Stafleu, F.A. \& Cowan, R.S. (1988) Taxonomic Literature. Volume VII: W-Z. Bohn, Scheltema \& Holkema, Utrecht, and Dr W. Junk b.v., Publishers, The Hague, XXX pp.

Vellozo, J.M. da C. (1829) Florae fluminensis. Typographia Nationali, Rio de Janeiro, 352 pp.

Wallich, N. (1830) Plantae asiaticae rariores 1(4). Treuttel \& Würtz, London, pp. 63-84.

Wu, C.-Y. \& Hsuan, S.-J. (1977) Clinopodium. In: Wu, C.-Y. \& Li, H.-W. (Eds.) Flora Reipublicae Popularis Sinicae 66 (2). Academia Sinica, pp. 222-239. 